\title{
Robust generation of hemangioblastic progenitors from human embryonic stem cells
}

\author{
Shi-Jiang Lu ${ }^{1}$, \\ Chenme Luol, \\ Katherine H olton ${ }^{1}$, \\ Q iang Feng ${ }^{1}$, \\ Yordanka Ivanova ${ }^{1} \&$ \\ Robert Lanza ${ }^{1,2 \dagger}$ \\ ${ }^{\dagger}$ Author for correspondence \\ IAdvanced Cell Technology, \\ 381 Plantation Street, \\ Worcester, M A 01605, U SA \\ 2Tel.: +1 5087561212 \\ ext. 655 ; \\ Fax: +1 508756 4468; \\ E-mail: rlanza@ \\ advancedcell.com
}

\begin{abstract}
Background: Human embryonic stem cells (hESCs) are a potentially inexhaustible source of cells for replacement therapy. However, successful preclinical and clinical progress requires efficient and controlled differentiation towards the specific differentiated cell fate. Methods: We previously developed a strategy to generate blast cells (BCs) from hESCs that were capable of differentiating into vascular structures as well as into all hematopoietic cell lineages. Although the BCs were shown to repair damaged vasculature in multiple animal models, the large-scale generation of cells under these conditions was challenging. Here we report a simpler and more efficient method for robust generation of hemangioblastic progenitors. Results: In addition to eliminating several expensive factors that are unnecessary, we demonstrate that bone morphogenetic protein (BM P)- 4 and VEGF are necessary and sufficient to induce hemangioblastic commitment and development from hESCs during early stages of differentiation. BMP-4 and VEGF significantly upregulate T-brachyury, KDR, CD31 and Lmo2 gene expression, while dramatically downregulating Oct-4 expression. The addition of basic FGF during growth and expansion was found to further enhance BC development, consistently generating approximately $1 \times 10^{8} \mathrm{BCs}$ from one six well plate of hESCs. Conclusion: This new method represents a significantly improved system for generating hemangioblasts from hESCs, and although simplified, results in an eightfold increase in cell yield.
\end{abstract}

$\mathrm{H}$ emangioblasts are bipotential progenitors that have the capacity to differentiate into both hematopoietic and endothelial cells [1]. The ability to generate an unlimited supply of these cells from human embryonic stem cells (hESCs) could have important clinical ramifications. We previously showed that $\mathrm{hESC}$-derived hemangioblasts homed to the site of injury and showed robust reparative function of damaged vasculature when injected into animals with diabetes or ischemia/reperfusion injury of the retina [2]. The cells also showed a similar regenerative capacity in animal models of both myocardial infarction ( $50 \%$ reduction in mortality rate) and hind-limb ischemia, with restoration of blood flow to near normal levels within a month. H owever, to realize their clinical potential, whether for vascular repair or various hematopoietic applications, it will be necessary to develop economical conditions for the robust and efficient differentiation of hemangioblastic progenitors from $\mathrm{hESC}$ s. For clinical application, the cells will also need to be animal and human pathogen free, generated in the absence of serum and stromal cells.

Several groups have generated hemangioblasts using hESC differentiation systems. Wang et al. demonstrated that $C D 45^{\text {neg }}$ PECAM $-1^{+}$, FLK $-1^{+}$and VE-cadherin ${ }^{+}$(CD 45 neg PFV) cells, derived from embryoid bodies (EBS) cultured in fetal bovine serum (FBS) and a mixture of growth factors and cytokines, possessed hemangioblast properties [3]. H owever, the rarity of the cells, less than a fraction of a percent of the cells, and the presence of FBS in the differentiation system precludes the possibility of its clinical use. Similarly, Zambidis et al. demonstrated the formation of multipotential mesodermal-hematoendothelial colonies using an FBS-containing culture system, although it is unclear whether these colonies can be expanded and/or whether they possess any functional activity in vivo [4]. Recently Kennedy et al. identified a human hemangioblast cell population derived from hESCs using a procedure that consisted of serum-free differentiation in a mixture of cytokines followed by expansion in serum-containing media [5]. H owever, all these studies utilize hESC s cultured on feeder cells and differentiated in FBS-containing medium. Importantly, to date, no other system has been described for the largescale generation or functional assessment of hESC - derived hemangioblasts.

We previously reported a two-step approach to generate blast cells (BC) from hESC $s$ without the use of serum [2]. These cells expressed gene signatures characteristic of hemangioblasts and 
clonally differentiated into multiple (erythroid, myeloid and multi-lineage) hematopoietic cell lineages as well as endothelial cells that expressed von Willebrand factor, CD 31/PECAM-1 and VE-cadherin. When the cells were injected into animals with vascular injury, they homed to the site of injury and showed robust reparative function of the damaged vasculatures [2]. In the present study, we investigated the roles of several factors in promoting differentiation of hESC $s$ toward hemangioblasts. We found that bone morphogenetic protein (BM P)-4, a ventral mesoderm inducer, is critical in hemangioblastic specification of hESC s, but not in the growth of $B C S$. VEGF, a multifunctional cytokine that plays a prominent role in normal vascular biology, is essential for efficient hemangioblastic specification of hESC s as well as the growth and expansion of $B C$ s in this serum-free system. The addition of basic FGF (bFGF) throughout the $B C$ growth and expansion stages, but not during $E B$ formation (hemangioblastic specification of $h E S C s)$, also enhanced the generation of $B C s$ significantly. D espite eliminating several expensive factors, including stem cell factor (SCF), Flt3-ligand (FL) and Tpo, these new improvements significantly optimize the generation of hemangioblasts from hESCs. In addition, we showed that feeder-free $\mathrm{hESC} s$ robustly generated hemangioblasts under these conditions; the development of this serum- and stromal-free system is important for clinical applications.

\section{Materials \& methods}

Culture of hESCS

hESC lines WAO1(H1), HUES3 and MA01 were used and maintained as previously described [6]. Briefly, hESCs were grown on mitomycin $\mathrm{C}$-treated mouse embryonic fibroblast (MEF) in complete hESC media. The hESC S were passaged every 3-5 days before reaching confluence using $0.05 \%$ trypsin- $0.53 \mathrm{mM}$ EDTA. For feeder-free culture, the cells were then grown on hESC -qualified $M$ atrigel $^{\mathrm{TM}}$ matrix (BD Biosciences, Bedford, MA, USA) in complete modified TeSR $^{T^{M}} 1$ (mTeSR ${ }^{T M} 1$ ) medium (Stem Cell Technologies, Inc, Vancouver, BC, Canada), which is based on the formulation of Ludwig et al. [7,8]. Cells were maintained according to manufacture's suggested instructions. Briefly, cells were passaged when they reached approximately $90 \%$ confluence, usually every 5-7 days with split ratios ranging from $1: 3$ to $1: 6$. Cells were treated with dispase (1 mg/ml, BD Biosciences) and incubated for $3-5 \mathrm{~min}$ at $37^{\circ} \mathrm{C}$ to begin dislodging the colonies. Colonies were washed with D M EM /F12 (M ediatech, M anassas, VA, USA) to remove dispase solution. To extricate the colonies from the tissue culture plastic, the wells were coated with D M EM /F12 and gently scraped until all of the colonies had been displaced. The colonies were transferred to conical tubes, the wells were washed with DMEM/F12 and the cells pooled to collect any remaining in the wells. They were centrifuged for $5 \mathrm{~min}$ at $1000 \mathrm{rpm}$. The cell pellets were resuspended in $\mathrm{mTeSR}^{\mathrm{TM}} 1$ media and transferred to $\mathrm{M}$ atrigel-coated six-well plates, in $2 \mathrm{ml}$ of $\mathrm{mTeSR}^{\mathrm{TM}} 1$ media per well. Cells were maintained at $37^{\circ} \mathrm{C}$ under $5 \% \mathrm{CO}_{2}$ and the $\mathrm{mTeSR}^{\mathrm{TM}} 1$ medium was replenished daily.

Immunoflourescent cytochemistry analysis Feeder-free $\mathrm{hESC}$ colonies were assayed for $0 \mathrm{ct}-4$ and Tra-1-60 expression using immunofluorescence. The cells werefixed with $4 \%$ paraformaldehyde, washed with phosphate-buffered saline (PBS), and blocked with $5 \%$ normal goat serum (Vector Labs, Burlingame, CA, USA), 1\% bovine serum albumin (Sigma, St Louis, M O, USA) and $0.2 \%$ Triton-X-100 (Sigma) in PBS for 30 min at room temperature. Cells were incubated with primary antibodies against $\mathrm{O}$ ct-4 (Santa Cruz Biotechnology, Santa cruz, CA, USA) or Tra-1-60 (M illipore/Chemicon, Temecula, CA, USA), in blocking solution, overnight at $4^{\circ} \mathrm{C}$, washed with PBS and incubated with a biotin conjugated secondary antibody (Jackson ImmunoResearch Labs, West Grove, PA, USA), in blocking solution, for $45 \mathrm{~min}$ at room temperature. After further washing, cells were incubated with Alexa 954 conjugated streptavidin (Invitrogen/M olecular probes, C arlsbad, CA, U SA), for 15 min at room temp followed by an extended final wash in PBS. Cells were mounted in Prolong G old with DAPI (Invitrogen/M olecular Probes).

\section{Differentiation of hemangioblasts \\ from hESCS}

To induce hESCs cultured on MEFs into hemangioblasts, $80-90 \%$ confluent plates were dissociated by $0.05 \%$ trypsin digestion. To differentiate feeder-free hESCs into hemangioblasts, $85-90 \%$ confluent cells were dislodged from the $M$ atrigel matrix using the protocol described earlier. Cells from both conditions were plated on ultra-low dishes (C orning, NY, USA) in Stemline II (Sigma) medium with different doses of BM P-4, VEGF and bFGF as described previously [2]. $\mathrm{H}$ alf of the medium was 
replaced after $48 \mathrm{~h}$ with fresh medium containing the same cytokines or the same medium plus SCF, FL and T po $(20 \mathrm{ng} / \mathrm{ml}$, R\& D System, Inc., M inneapolis, M N , USA), which depend on different experiment conditions. After 3.5 days, EBs were collected and dissociated by $0.05 \%$ trypsin. Singlecell suspensions were obtained by passing the cells through 22-gauge needle and through a $40-\mu \mathrm{m}$ cell strainer, collected by centrifugation and resuspended in $50-100 \mu$ of Stemline II media. Cells $\left(0.75 \times 10^{5}\right.$ to $\left.1 \times 10^{5}\right)$ were mixed with $2.5 \mathrm{ml}$ of blast colony growth medium (BGM) as previously described [2], plated in ultra-low dishes and incubated at $37^{\circ} \mathrm{C}$. Blast colonies derived from both MEF and feeder-free hESCs were observed 3-4 days after plating, followed shortly thereafter by rapid expansion. BC $s$ are defined in the current study as cells obtained from day- 6 blast colonies.

Enrichment of hemangioblast precursors Potential $B C$ precursor surface markers $C D 31$, CD 34, KDR, CXCR-4, CD 133, ACE, PCLP1, PDGFR $\alpha$, Tie-2, N rp-2, Tpo-R and bFGFR-1 were selected for cell enrichment. All antibodies are mouse monoclonal IgG isotype and they are: CD 31 and CD 34 (D ako Cytomation, Carpinteria, $C A, U S A), K D R$ and $T p o-R$ ( $R \& D$ Systems, Inc.), CXCR-4 (Abcam Inc., C ambridge, M A, USA), N rp-2, ACE, PCLP1 and PD GFR $\alpha$ (Santa Cruz Biotechnology), Tie2 (Cell Signaling Technology, Inc., Danvers, MA, USA), bFGFR-1 (Zymed Laboratories, San Francisco, CA, USA) and CD133 (Miltenyi Biotech, Auburn, CA, USA). Antibody cocktail assembly was performed by EasySep 'D o-it-Yourself' Selection Kit (Stem C ell Technologies). Cell suspensions derived from EBs were centrifuged at $1200 \mathrm{rpm}$ for $4 \mathrm{~min}$ and resuspended in PBS with $2 \% \mathrm{FBS} / 1 \mathrm{mM}$ EDTA buffer at a concentration of $1-2 \times 10^{6}$ cells/100 $\mu$ l. The cells were mixed with different antibody cocktails for $15 \mathrm{~min}$ at room temperature and then incubated with EasySep $\mathrm{N}$ anoparticle at room temperature for 10 additional minutes. Positive selected cells were separated after pouring off supernatant when placing tube with cells in a $\mathrm{M}$ agnet holder. Antibody selected positive cells $\left(1 \times 10^{5}\right)$ were mixed with $2.5 \mathrm{ml}$ of BGM and plated for blast colony development.

Real-time RT-PCR \& data analysis

Total RN A was extracted from EBs or undifferentiated $h E S C$ s using $R N$ easy $M$ icro Kits ( $Q$ iagen, Valencia, CA, USA) according to manufacturer's protocol. CDNAs were synthesized using BD SM ART PCR CD N A Synthesis $K$ it (BD Biosciences) per manual instructions. Real-time RT-PCR (qRT-PCR) was performed using FullVelocity SYBR Green QPCR M aster M ix (Stratagene, La Jolla, CA, USA). The reactions were set up in triplicate with the following components per reaction: $50 \mathrm{ng}$ of template, 0.2 $\mu \mathrm{m}$ of each primer and $1 \mathrm{X}$ M aster mix. Genespecific sequences of the primers used are listed in Table1, and annealing temperature for all primers is $55^{\circ} \mathrm{C}$. Amplification and real-time data acquisition were performed in a Stratagene $M \times 3005 P$ with $M$ xPro version 3.0 software. The following cycle conditions were used: one cycle of $95^{\circ} \mathrm{C}$ for $10 \mathrm{~min}$ followed by 40 cycles of $95^{\circ} \mathrm{C}$ for $30 \mathrm{~s}, 55^{\circ} \mathrm{C}$ for $1 \mathrm{~min}, 72^{\circ} \mathrm{C}$ for $30 \mathrm{~s}$ followed by a final cycle of $95^{\circ} \mathrm{C}$ for $1 \mathrm{~min}, 55^{\circ} \mathrm{C}$ for $30 \mathrm{~s}$ and $95^{\circ} \mathrm{C}$ for $30 \mathrm{~s}$. Relative quantification of each target gene was performed based on cycle threshold $\left(C_{T}\right)$ normalization to $\beta$-actin $\left(\Delta \mathrm{C}_{\mathrm{T}}\right)$ using the $\Delta \Delta \mathrm{C}_{\mathrm{T}}$ method [9]. Analysis of relative gene-expression data using real-time quantitative $P C R$ and the $2(-\Delta \Delta(T))$ method [9], where the $\Delta C_{T}$ of each examined gene in the experimental samples was compared with average $\Delta \mathrm{C}_{\mathrm{T}}$ of each gene in an undifferentiated $\mathrm{hESC}$ control

Table 1. Sequences of gene-specific primers used in real-time RT-PCR.

\begin{tabular}{|c|c|c|c|}
\hline Gene & Forw ard primer $\left(5^{\prime} \rightarrow 3^{\prime}\right)$ & Reverse primer $\left(5^{\prime} \rightarrow 3^{\prime}\right)$ & Ref. \\
\hline OCT-4 & GAAGGTATTCAGCCAAACGC & GTTACAGAACCACACTCGGA & \\
\hline BRACH & TGCTTCССTGAGACCCAGTT & GATCACTTCTTCCTIGGCATCAAG & [33] \\
\hline MixL1 & CCGAGTCCAGGATCCAGGTA & CTCTGACGCCGAGACTTGG & [33] \\
\hline KDR/Flk1 & CCAGCCAAGCTGTCTCAGT & CTGCATGTCAGGTTGCAAAG & [4] \\
\hline CD31 & GAGTCCTGCTGACCCTTCTG & ATTTGGCACCGTCCAGTCC & [4] \\
\hline $\mathrm{SCl} / \mathrm{TAL1}$ & ATGAGATGGAGATTACTGATG & GCCCCGTTCACATTCTGCT & [4] \\
\hline LMO2 & AACTGGGCCGGAAGCTCT & CTTGAAACATTCCAGGTGATACA & [4] \\
\hline GAPDH & CGATGCTGGCGCTGAGTAC & CCACCACTGACACGTTGGC & \\
\hline$\beta$-actin & GCGGGAAATCGTGCGTGACA & GATGGAGTTGAAGGTAGTTCG & \\
\hline
\end{tabular}


Figure 1. Effects of bone morphogenetic proteins and VEGF $F_{165}$ on the development of blast colonies.

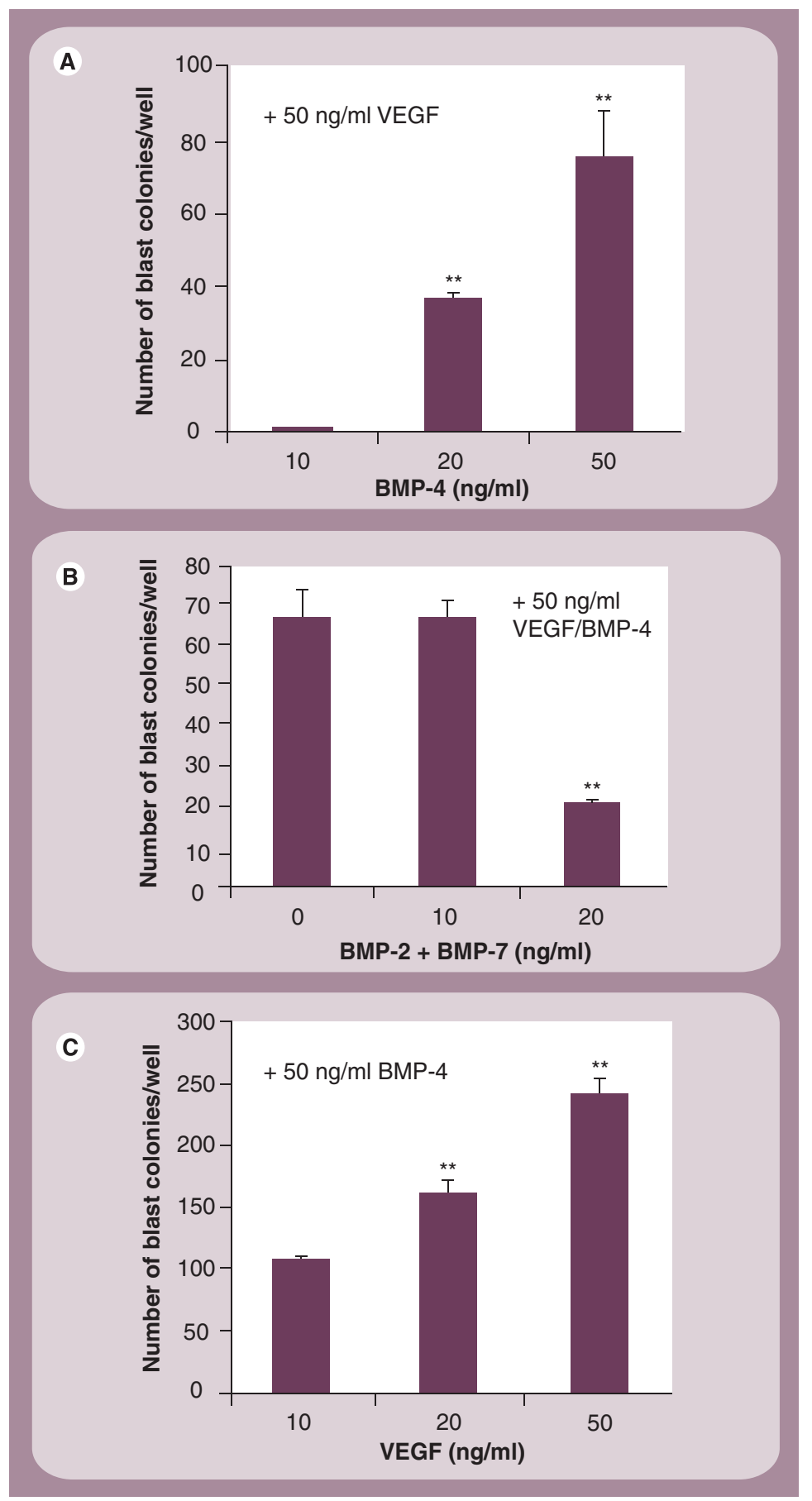

(A) Different doses of BM P-4 were added in embryoid body (EB) medium containing $50 \mathrm{ng} / \mathrm{ml}$ of $V_{E G F}$, and a dose-dependent development of blast colonies was observed for BM P-4. (B) EB medium containing $50 \mathrm{ng} / \mathrm{ml}$ of BM P-4 and VEGF $F_{165}$ were supplemented with different doses $(0,10$ and $20 \mathrm{ng} / \mathrm{ml})$ of BM P-2 and -7 . BM P-2 and -7 failed in promoting blast colony development. (C) Different doses of VEGF $_{165}$ were added in EB medium containing $50 \mathrm{ng} / \mathrm{ml}$ of BM P-4. The development of blast colonies is VEGF 165 dose dependent.

$* * p<0.01, n=3.1 \times 10^{5}$ cells from day 3.5 EBs were plated per well. BMP: Bone morphogenetic protein. sample $\left(\Delta \Delta \mathrm{C}_{T}\right)$. Then the fold change in expression was calculated as $2-\mathrm{h} \Delta \Delta^{\mathrm{CT}}$. The negative fold difference data was convert to a linear 'fold change in expression' value using the following formula: linear fold change in expression $=$ -(1/fold change in expression).

\section{Statistical analysis}

All data were presented as mean \pm SEM . Intergroup comparisons were performed by unpaired Student's t-test using GraphPad Prism, version 4, software (GraphPad Software, Inc., San Diego, CA, USA). $p<0.05$ was interpreted as statistically significant.

\section{Results}

Both BMP-4 \& VEGFs are required for hemangioblast development

We previously described a serum-free system to induce hESC differentiation toward the hemangioblastic and hematopoietic lineages $[2,10]$. Although BM P-4, VEGF and a cocktail of early hematopoietic cytokines were used, the absolute requirement and optimal concentrations of the individual factors were not examined. In order to reduce the expense and effort necessary to generate hemangioblasts for future research and clinical applications, we specifically examined the minimal requirements and effects of VEGFs, BM Ps and three early hematopoietic cytokines (TPO, FL and SCF) on the efficient development of blast colonies from hESCs. We found that BM P-4 is absolutely required for the development of blast colonies under serum-free conditions. $\mathrm{N}$ o blast colonies were obtained without the supplement of BPM -4 in the medium during $E B$ formation and a clear dose-response effect of BM P-4 was observed for the formation of blast colonies from hESCS (Figre1A). Furthermore, we found that BM P-4 could not be substituted by other members of the BM P family. BM P-2 and -7 alone, or a combination of the two, failed to promote $B C$ development. Furthermore, supplementation of BMP-2 and -7 in EB medium containing BM P-4, either showed no effect $(10 \mathrm{ng} / \mathrm{ml})$ or inhibited $(20 \mathrm{ng} / \mathrm{ml})$ blast colony development (Figure 1B). H owever, addition of BM P-4, and BM P-2 and/or BM P-7 in $B G M$ did not have any effect on the development of blast colonies, suggesting that BM P-4 only promotes the mesoderm/hemangioblastic specification stage, but not the growth and expansion of BCS. Similarly, no blast colonies developed when $V E G F_{165}$ was eliminated from the EB formation medium. VEG $F_{165}$ was found 
Figure 2. The effect of basic FGF on the development of blast colonies added during different stages.

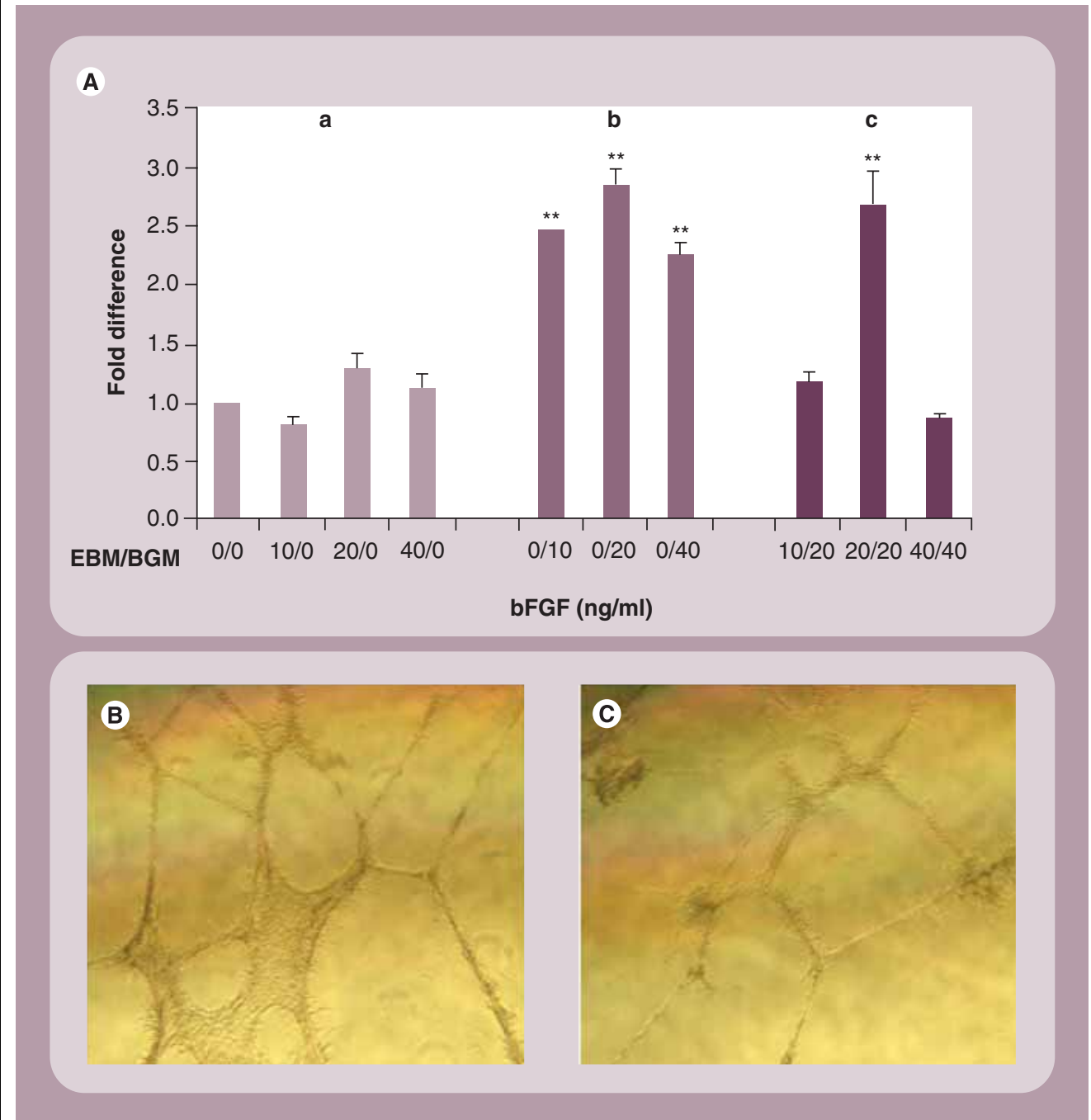

(A-a) Different doses of bFGF were added in EBM ; (A-b) Different doses of bFGF were supplemented in BGM ; (A-C) Different doses of bFGF were added in both EBM and BGM. **p $<0.01, n=3$. (B \& C) Networklike structure formation of endothelial cells derived from blast cells developed in BGM with (B)

and without (C) bFGF. Endothelial cells from both sources formed network-like structures with no obvious difference.

bFGF: Basic FGF; BGM : Blast colony growth medium; EBM : Embryoid body medium.

to promote the development of blast colonies in a dose-dependent manner (Figre1C). VEG $F_{121}$, an isoform of VEGF members that can only bind to KDR and FLT 1 receptors [11], can be used as a substitute of VEGF $\mathrm{F}_{165}$ in promoting the development of blast colonies from hESCs; almost identical numbers of blast colonies (68 \pm 5 vs $67 \pm 12$ ) were developed when $50 \mathrm{ng} / \mathrm{ml}$ of either VEGF $F_{165}$ or VEGF $F_{121}$, which is the optimal dose under serum-free condition, was added in EB medium. H owever, in contrast to BMP-4, no blast colonies were obtained if
VEGF was absent in BGM , demonstrating that VEG F plays a critical role both in early stage of mesoderm/hemangioblastic specification and in the growth and expansion of $\mathrm{BC}$.

In our original report [2], TPO, FL and SCF were added $48 \mathrm{~h}$ after plating hESC $s$ in EB medium in an effort to further promote early hematopoeitic progenitor growth and expansion. $\mathrm{H}$ ere we examined whether TPO, FL and SCF played any role in the specification of $h E S C S$ toward the mesoderm/hemangioblast lineage. EBs were formed by plating hESCs in Stemline II 
Figure 3. The effect of basic FGF on the development of blast colonies from three human embryonic stem cell lines.

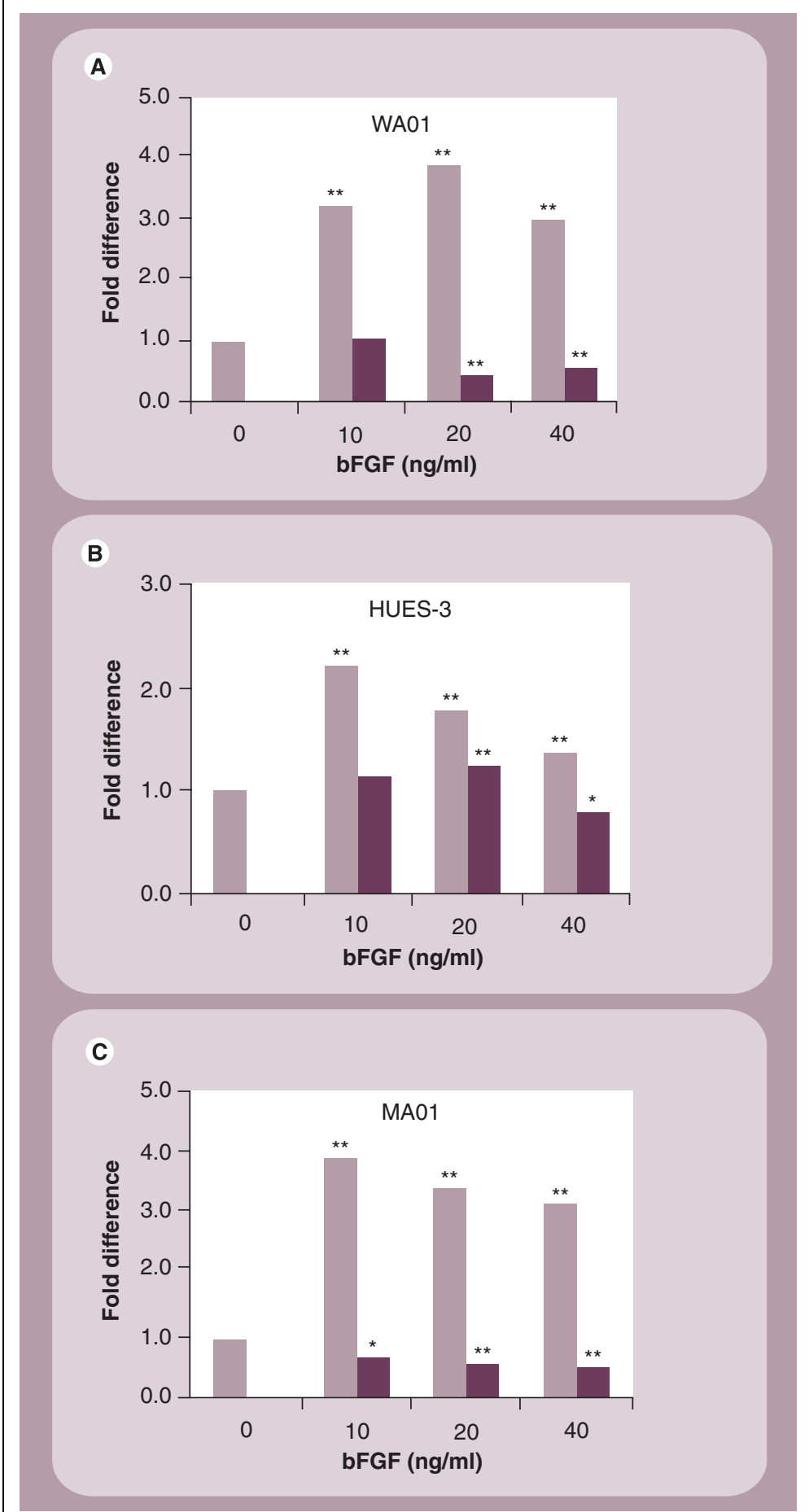

Light gray: Different doses of bFGF were added in blast colony growth medium. Dark gray: Various doses of bFGF were added in embryoid body medium.

$* \mathrm{p}<0.05 ; * * \mathrm{p}<0.01 ; \mathrm{n}=3$.

bFGF: Basic FGF. other well, no additional factor was added, and the EBs were incubated for another $36 \mathrm{~h}$. EBs were then collected and single cell suspension was obtained and plated for blast colony formation. $O$ ur results show that supplement of TPO, FL and SCF during EB formation has no effect on the development of blast colonies, $242 \pm 16$ versus $287 \pm 33$ blast colonies developed per $1 \times 10^{5}$ cells derived from EBs treated with and without $T P O, F L$ and $S C F$, respectively. $\mathrm{H}$ owever, both TPO and FL are required for blast colony growth and expansion.

bFGF promotes the growth, but not commitment, of hemangioblasts from hESCS

Previous studies have shown that supplement of bFGF during early differentiation promotes murine and human ESC hematopoietic development $[5,12-14]$. Thus, we investigated whether the addition of bFGF during the EB differentiation stage would enhance blast colony formation from $\mathrm{hESC}$. Addition of bFGF during EB formation had no effect on the development of blast colonies, and, in fact, at a higher dose $(40 \mathrm{ng} / \mathrm{ml})$ inhibited the formation of blast colonies from multiple hESC lines (Figures 2A \& 3). By contrast, the addition of bFGF in BGM significantly enhanced the development of blast colonies (Figures2A \& 3). Both the number of blast colonies $(p<0.001)$ and total number of BCs increased significantly compared with BGM without bFGF supplementation. With bFGF at optimal dose $(20 \mathrm{ng} / \mathrm{ml})$ in $B G M$, the blast colonies are larger and healthier, and we consistently harvest approximately $1 \times 10^{8} \mathrm{BCs}$ from one six-well plate of high-quality WAO1 hESCs (approximately $1.2 \times 10^{7}$ cells) after 6 days growth, which is $8 \pm 1$-fold higher than that obtained from BGM without the supplement of bFGF.

To investigate the lineage differentiation potentials of $B C s$ generated with and without supplementation of bFGF, equal numbers of pooled BCs were plated for hematopoietic and endothelial lineage differentiation as previously described [2]. For hematopoietic CFU formation, $129 \pm 9$ and $86 \pm 22$ CFU s $/ 10^{4}$ BCs were formed from BCs derived from $B G M S$ supplemented with and without bFGF $(20 \mathrm{ng} / \mathrm{ml})$, respectively. Furthermore, no difference was observed for the development of different CFUs (CFU-mix, CFU-G, CFU-M and (FU-E) between the two groups (data not shown). For endothelial lineage differentiation, more BCs $(62 \pm 3 \%)$ from BGM with bFGF $(20 \mathrm{ng} / \mathrm{ml})$ differentiated into endothelial 
cells than BCS $(55 \pm 3 \%)$ derived from BGM without bFGF supplement. Endothelial cells from both sources formed capillary vascular-like structures efficiently after plating on $M$ atrigel (Figre2B $\boldsymbol{\&} \mathbf{C})$. These results suggest that bFGF promotes the growth of $\mathrm{BCS}$, but does not cause preferential lineage differentiation.

Robust generation of hemangioblasts from hESCs maintained without feeder cells

It has been reported that $\mathrm{hESC} s$ maintained on MEF feeders contain the nonhuman sialic acid $\mathrm{N}$-glycolylneuraminic acid (N eu5G c) [7,8,15], and that animal sources of $\mathrm{N}$ eu $5 \mathrm{G} \mathrm{c}$ can cause a potential immunogenic reaction with human complement. The culturing of hESC $s$ on MEF feeder layers prevents complete elimination of animal $\mathrm{Neu5G} \mathrm{c}$, and raises concerns for the potential clinical applications of hemangioblasts generated from hESC lines maintained under these conditions. Therefore, we have taken steps to determine whether hemangioblasts can be generated from hESCs maintained without MEF feeders. Three hESC lines were passaged with dispase onto plates coated with $\mathrm{hESC}$-qualified $M$ atrigel matrix, and maintained in mTeSR medium as described in $M$ aterials \& methods. Their undifferentiated state was confirmed with immunoflourescence staining for the expression of $0 \mathrm{ct}-4$ and Tra-1-60 antigens and colony morphology (Figre4A-H). These cells were collected and utilized for the development of $\mathrm{BC}$ s using the optimized conditions described earlier. Interestingly, a significantly higher number of BCs were observed with feeder-free hESCs as compared with hESC s cultured on M EF feeders when identical numbers of EB cells were plated (Figre 4I; $p<0.05)$. These results were observed for all three tested hESC lines WA01, M A01 and HUES-3 (data not shown).

Mechanism underlying the effects of BMP-4 \& VEGF on hemangioblast development In order to dissect the molecular mechanism underlying the effects of BM P-4 and VEGF on hemangioblast development from $h E S C S$, we compared the expression of genes associated with the development of hemangioblasts in 3.5-dayold EBs that were formed in Stemline II medium both with and without each factor, as well as with a combination of BM P-4 and VEGF. Gene expression was analyzed by qRT-PCR and compared with their levels in undifferentiated hESC s. EBs formed without any factor expressed higher levels of OCT-4, a marker for hESC $\mathrm{s}$, than undifferentiated $h E S C s$. Supplementation of VEGF in $E B$ medium led to a moderate downregulation of OCT-4 expression, whereas the addition of BM P-4 or BM P-4 plus VEGF resulted in a significant decrease in OCT-4 expression ( $p<0.0005$; Figre5). There was no additive effect of BM P-4 and VEGF on OCT-4 expression. The expression of $\mathrm{T}$-brachyury gene, the earliest marker expressed in mesoderm cells, was downregulated in all samples except EBs derived from cultures containing both BM P-4 and VEGF (the latter showing a significant increase in its expression; $p<0.0005)$. Similar expression patterns were observed for CD 31 and L mo2; significantly increased levels of expression were only detected in EBs exposed to a combination of BM P-4 and VEGF $(p<0.0005)$. KDR, one of the most studied VEGF receptor, has been shown to be expressed in all hESC lines [4,5]; its expression was dramatically downregulated in EBs derived from media with no addition of exogenousfactor, and with supplement of BM P-4 or VEG F alone. H owever, a moderate but significant increase in KDR expression was observed in EBs formed in the presence of BM P-4 and VEGF $(p<0.002)$, a condition that promoted efficient development of hemangioblasts from hESC s. Surprisingly, in contrast to a recent report [14], substantial decreases in the expression of M ixL-1 and SCL/TAL-1 genes were detected in EBs formed in all conditions. O ne possible explanation is that growth in different serum-free media caused a different expression pattern in these genes. $\mathrm{N}$ evertheless, these results suggest that the commitment and development of mesoderm/hemangioblast from hESCs requires both BM P-4 and VEG F, consistent with the results of blast colony development (Figre1).

Identification of surface markers for progenitors of blast cells

In our original method [2], BC s were generated by replating day $3.5 \mathrm{EBscells}$ in $1 \%$ methylcellulose supplemented with defined factors. This strategy is important when identifying BCs that possess the potential to form hematopoietic and endothelial cells, and it is also reproducible when generating $B C s$ from hESCs. However, this approach utilizes dishes in standard tissue culture incubators, and thus cannot be adapted to rotary bioreactors for scale-up. This limitation is mainly due to the fact that cells from day 3.5 $E B s$ are heterogeneous and include undifferentiated hESC s (only a portion of the cells are BC 
Figure 4. Human embryonic stem cells grown under feeder-free conditions retain pluripotency markers and are capable of robust hemangioblast differentiation.

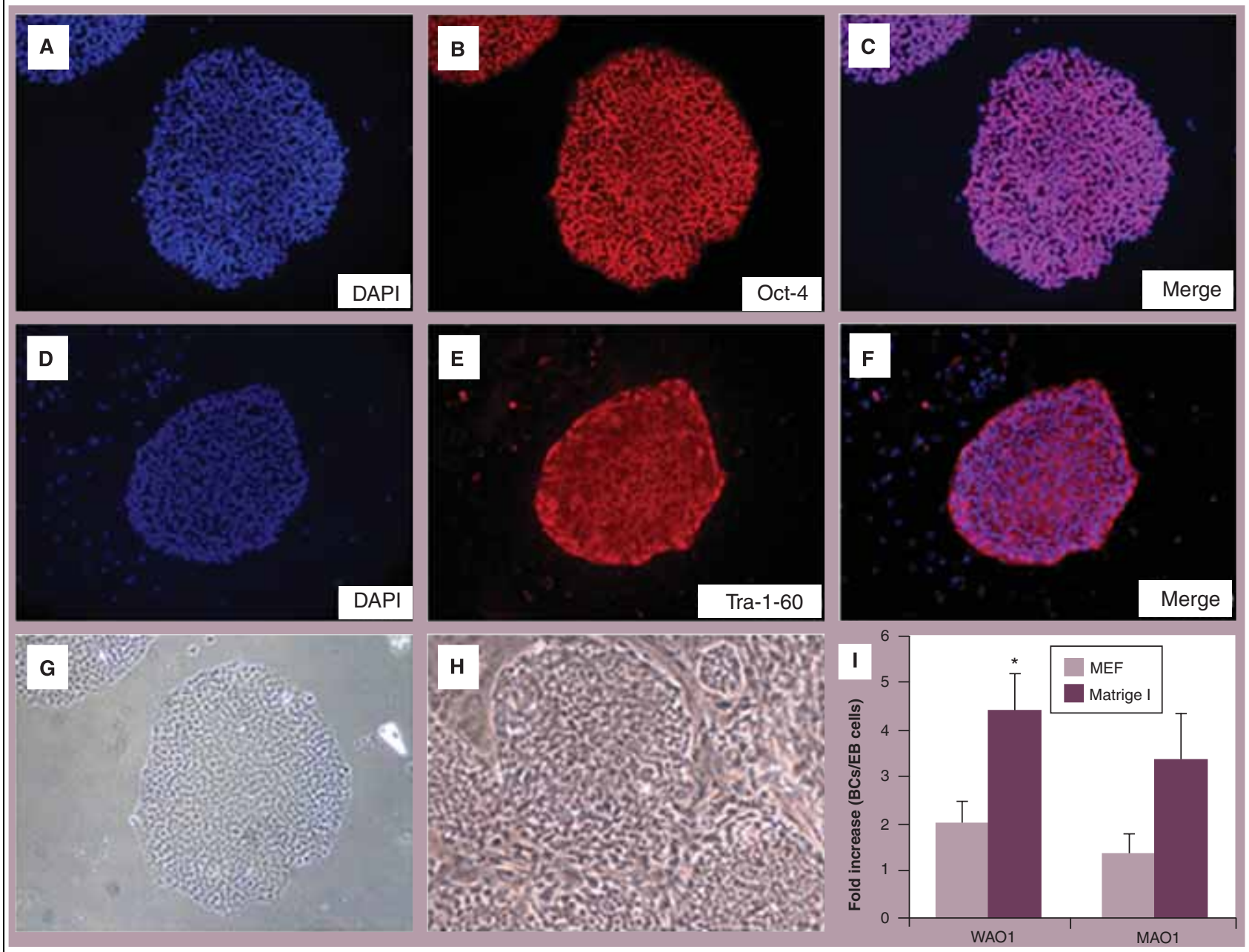

After four to five passages under feeder-free conditions WA01 cells were stained for expression of the human embryonic stem cell (hESC) markers Oct-4 ([A-C] DAPI, Oct-4 and merged, respectively) and Tra-1-60 ([D-F] DAPI, TRA-1-60 and merged, respectively).

$(\mathbf{G} \& \mathbf{H})$ demonstrate differences in colony morphology when $\mathrm{hESC}$ s are cultured on Matrigel (G) versus M EFs (H). Magnification $\times 100$.

(I) hESCs were grown either on MEFS or M atrigel and then differentiated under the optimized conditions described in $M$ aterial \& methods. Considerably more hemangioblast expansion was observed in Matrigel cultured cells as compared with MEF cultured hESCs.

$* \mathrm{p}<0.03 ; \mathrm{n}=3$.

BC: Blast cell; EB: Embryoid body; MEF: Mouse embryonic fibroblast.

progenitors). Replating this heterogeneous population in liquid culture would therefore lead to the growth of all cells including the formation of secondary EBs from undifferentiated hESC s, excluding their possible use in clinical applications. H owever, if a marker(s) for the progenitor of $\mathrm{BC} s$ can be identified, the purified progenitor can be seeded in liquid culture adapted with a rotary bioreactor for scaled-up production of BCS. We therefore selected 12 cell surface molecules that are associated with the development of mesoderm derivatives. The corresponding antibodies were used to enrich cells from day $3.5 \mathrm{EBs}$, and the enriched cells assayed for blast colony-forming ability. As shown in Figre6, $K D R^{+}$cells from 3.5-day EBs generated threetimes more blast colonies than the unfractioned control cells $(p<0.01)$, which is consistent with previous studies [5]. Although we also found a moderate increase in blast colonies ( 1.5-fold) after plating CD $31^{+-}$and $C D 34^{+}-$enriched populations, the increase did not reach statistical significance. All other enriched populations produced equal or less blast colonies as compared with unfractioned control cells, indicating that the BC progenitor does not express these molecules. The unbound (flow 
through) cells of all antibodies tested al so formed similar numbers of blast colonies as the unfractioned cells, suggesting that even KD R+, CD $34^{+}$ and $C D 31^{+}$cells represent a very limited portion of the cells that are capable of forming blast colonies. 0 wing to the limitation of the EasySep 'D oit-Yourself' Selection Kit used in the current study, double positive cells were not examined.

\section{Discussion}

We describe a significantly improved system for generating hemangioblasts from hESCs. Although simplified, this new method results in an $8 \pm 1$-fold increase in $B C$ yield. 0 ur data also show that hESC specification and commitment to the hemangioblastic lineage is promoted very efficiently by only two factors, BMP-4 and VEGF, during EB formation in Stemline II medium. The supplement of low-dose bFGF in BGM further increases the efficiency and yield of $B C$ s. This two-step system does not require the use of different cytokine combinations at multiple steps, nor does it require serum or conditioned medium as reported by Kennedy et al. [5], which could contribute to wide variations in efficiency and reproducibility. Furthermore, the elimination of several factors, including serum and animal feeder cells from our system, as well as the ability to generate significantly larger number of BCs should be desirable for future preclinical and human studies both in terms of time and cost.

BM P-4, one of the well-defined members of the BM $P$ family, has been shown to play a pivotal role in the patterning of embryonic ventral mesoderm and the formation of precursors for hematopoietic and endothelial cells in both murine and primate ESC differentiation systems $[5,14,16-23]$. VEGF is a multifunctional cytokine that has also been shown to play a important role in hematopoietic differentiation of ESCS $[5,13,14,20,21,24]$. O ur data show that both BM P-4 and VEGF are necessary and sufficient to efficiently induce hemangioblastic commitment of hESC $s$ during the early stages of differentiation under serum-free conditions. The absence of either factor during $E B$ formation prevents development of blast colonies. G ene-expression analyses confirmed that the upregulation of KDR, CD 31, T-brachyury and Lmo2 genes required the presence of both $B M P-4$ and VEGF. The effect of BM P-4 was specific, and

\section{Figure 5. Real-time RT-PCR analysis of gene expression in embryoid bodies cultured under}

\section{different conditions.}

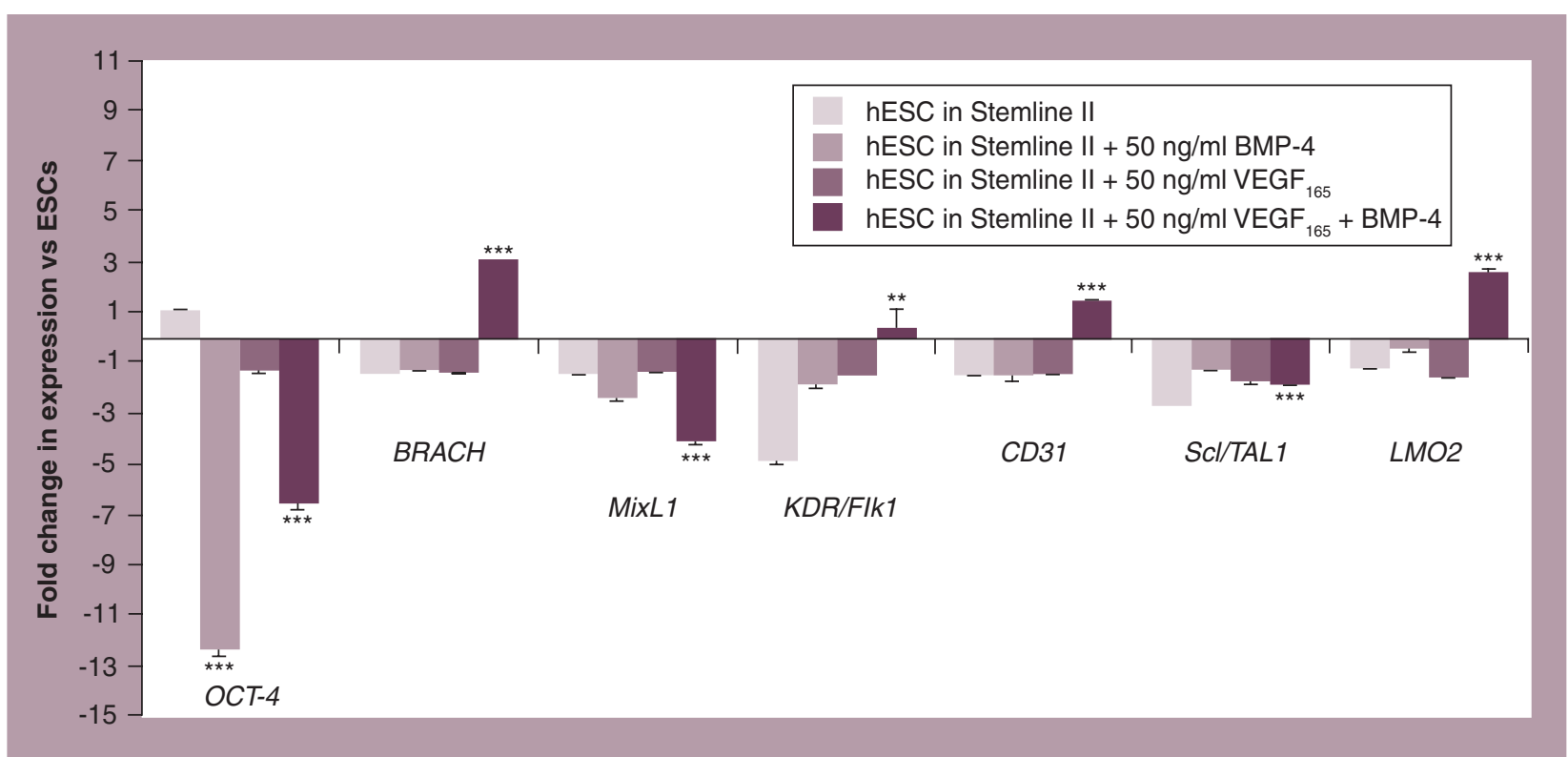

Expression levels of various genes associated with development of hemangioblasts were analyzed in embryoid bodies derived in the presence or absence of either or a combination of both bone morphogenetic protein- 4 and VEGF $F_{165}$. $\beta$-actin was used as an internal control to normalize gene expression. Relative gene expression is presented as a fold difference compared with average expression levels observed in undifferentiated human ESCs.

$* * \mathrm{p}<0.002 ; * * * \mathrm{p}<0.0004 ; \mathrm{n}=3$.

ESC: Embryonic stem cell. 


\section{Figure 6. Identification of surface markers for hemangioblast progenitors.}

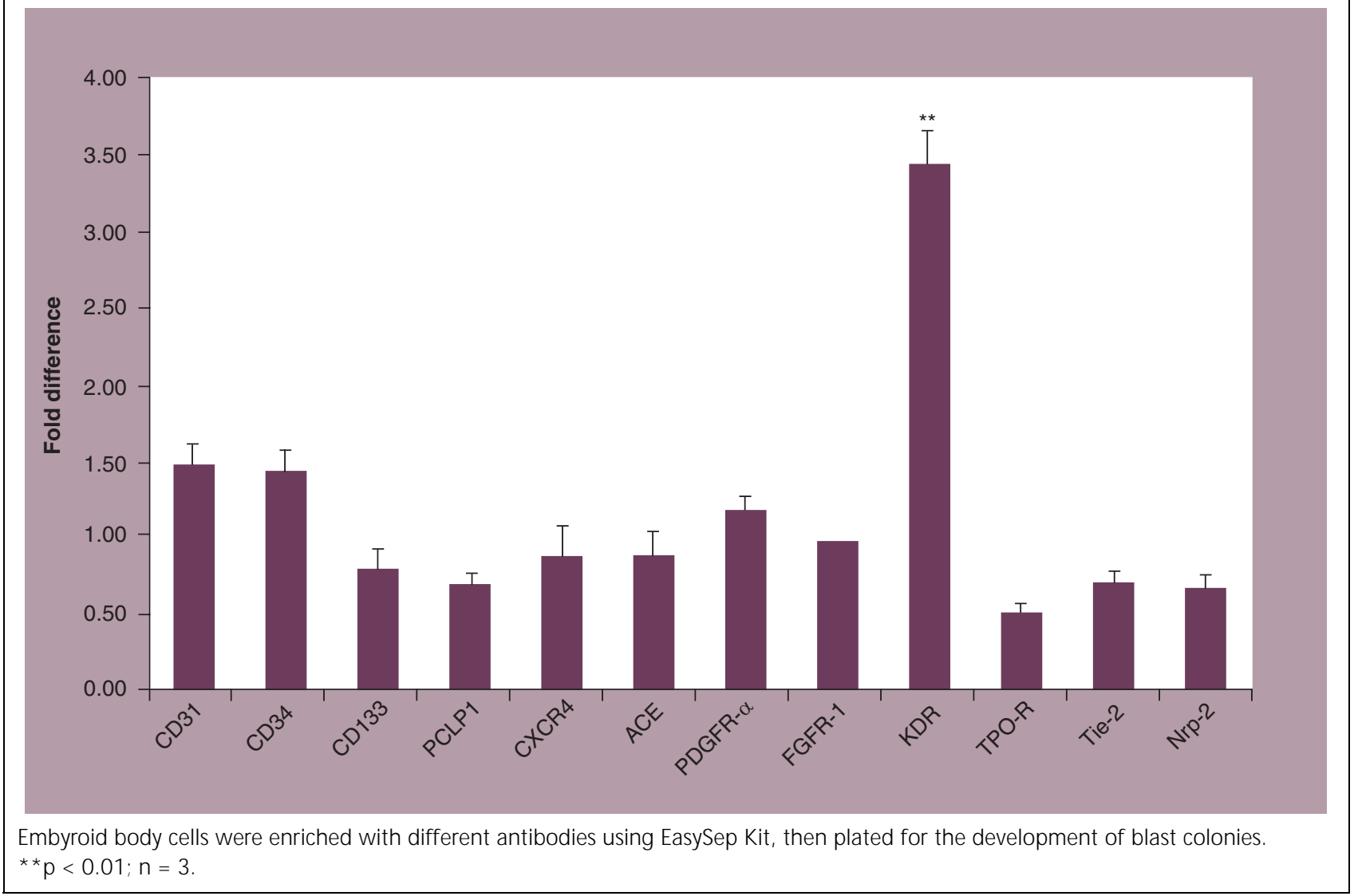

other members of the BM P family showed no effects on promoting blast colony formation. H owever, we found that VEGF 165 could be substituted by VEGF $\mathrm{F}_{121}$. The latter can only bind to KDR and FLT 1, but not NRP-1 and -2 receptors [11], consistent with observations in the mouse ESC system [25]. O ur progenitor cell enrichment results confirmed these results: $K D R^{+}$cells showed a significant increase in blast colony formation, whereas NRP-2+ cells revealed a lack of enhancement of blast colony development.

bFGF has been supplemented in culture medium to sustain $\mathrm{hESC}$ undifferentiated status and maintain their self-renewal capacity [26-28]. FG Fs have also been shown to play a role in the development of cardiomyocytes and preserve long-term repopulating hematopoietic stem cells in vitro [29-31]. Previous work demonstrated that bFGF induced hematopoietic and hemangioblastic specification when added to EB medium in both the mouse and human ESC differentiation systems [5,12-14]. $O$ ur results clearly demonstrated that addition of $\mathrm{bFGF}$ during $\mathrm{EB}$ formation failed to promote $B C$ development, consistent with the fact that FGFR + cells did not increase the number of blast colonies as compared with controls. However, bFGF in BGM significantly enhanced the growth and expansion of blast colonies, which is consistent with the observation of Kennedy et al. [5]. There are several possible explanations for the discrepancies, and presumably reflects different properties of mouse and human ESCS [32]. In addition, in hESC studies, Kennedy et al. demonstrated that a multistep procedure [5], BM P-4 followed by the addition of $b F G F$, then by $b F G F$ plus VEGF, resulted in the formation of hemangioblastic and hematopoietic progenitors. Pick et al. showed that a combination of four factors including bFGF in EB medium promoted hESC hematopoietic differentiation [14], but neither study addressed the precise role of the added factors. All of these studies, including our own, utilized different components that could have contributed to the observed discrepancies on the effects of bFGF.

In summary, we have developed a simple and efficient system for generating functional $B C s$ from human ESC s cultured with and without 
animal feeder cells. The efficient and controlled differentiation of $\mathrm{hESC}$ s into specific lineages in serum- and animal feeder-free systems is important for their productive use in regenerative medicine.

\section{Acknowledgements}

The authors are grateful to William $\mathrm{H}$ olmes for his help in setting up qRT-PCR and data analysis and Jennifer S Park for critical reading of the manuscript.
Financial \& competing interests disclosure

S-J Lu, C Luo, K H olton, Q Feng, Y Ivanova and R Lanza are employees of Advanced Cell Technology, a stem cell company in the field of regenerativemedicine. The authors have no other relevant affiliations or financial involvement with any organization or entity with a financial interest in or financial conflict with the subject matter or materials discussed in the manuscript apart from those disclosed.

$\mathrm{No}$ writing assistance was utilized in the production of this manuscript.

\section{Executive summary}

- We describe a significantly improved system for generating hemangioblasts from human embryonic stem cells (hESCs). Although simplified, this new method results in a near-exponential ( $8 \pm 1$-fold) increase in blast cell $(\mathrm{BC})$ yield.

- Our data show that hESC specification and commitment to the hemangioblastic lineage is promoted very efficiently by only two factors, bone morphogenetic protein (BM P)-4 and VEGF. The absence of either factor during embryoid body (EB) formation prevents development of blast colonies.

- Gene-expression analyses confirmed that the upregulation of KDR, CD31, T-brachyury and Lmo2 genes required the presence of both BM P-4 and VEGF. The effect of BM P-4 was specific, and other members of the BM P family showed no effects on promoting blast colony formation.

- This two-step system does not require the use of different cytokine combinations at multiple steps, nor does it require serum or conditioned medium as previously reported, which could contribute to wide variations in efficiency and reproducibility. The ability to generate significantly larger number of BCs should be desirable for future preclinical and human studies both in terms of time and cost.

- In conclusion, we have developed a simple and efficient system for generating functional BCs from hESCs cultured with and without animal feeder cells. The efficient and controlled differentiation of hESCs into specific lineages in serum- and animal feeder-free systems is important for their productive use in regenerative medicine.

\section{Bibliography}

1. Wagner RC: Endothelial cell embryology and growth. Adv. M icrocirc. 9, 45-75 (1980).

2. Lu SJ, Feng $Q, C$ aballero $S$ et al.: Generation of functional hemangioblasts from human embryonic stem cells. $N$ at. M ethods 4(6), 501-509 (2007).

3. Wang $L$, Li L, Shojaei $F$ et al.: Endothelial and hematopoietic cell fate of human embryonic stem cells originates from primitive endothelium with hemangioblastic properties. Immunity 21(1), 31-41 (2004).

4. Zambidis ET, Peault B, Park TS, Bunz F, Civin $\mathrm{Cl}$ : $\mathrm{H}$ ematopoietic differentiation of human embryonic stem cells progresses through sequential hematoendothelial, primitive, and definitive stages resembling human yolk sac development. Blood 106(3), 860-870 (2005).

5. Kennedy M, D 'Souza SL, Lynch-K attman M, Schwantz S, Keller G: $D$ evelopment of the hemangioblast defines the onset of hematopoiesis in human ES cell differentiation cultures. Blood 109(7), 2679-2687 (2007).
6. Klimanskaya I, M CM ahon J: Approaches of derivation and maintenance of human ES cells: detailed procedures and alternatives. In: $\mathrm{H}$ andbook of Stem Cells. Volume 1: Embryonic Stem Cells. Lanza R et al. (Eds.). Elsevier/Academic Press, N ew York, U SA 437-449 (2004).

7. Ludwig TE, Bergendahl V, Levenstein M E, Yu J, Probasco M D , Thomson JA: Feederindependent culture of human embryonic stem cells. $N$ at. M ethods 3(8), 637-646 (2006).

8. Ludwig TE, Levenstein M E, Jones JM et al.: D erivation of human embryonic stem cells in defined conditions. $\mathrm{N}$ at. Biotechnol. 24(2), 185-187 (2006).

9. Livak KJ, Schmittgen TD: Analysis of relative gene expression data using real-time quantitative $P C R$ and the $2(-\Delta \Delta C(T))$ method. M ethods 25(4), 402-408 (2001).

10. Lu SJ, Feng $Q$, Ivanova $Y$ et al.: Recombinant $\mathrm{H}$ oxB4 fusion proteins enhance hematopoietic differentiation of human embryonic stem cells. Stem Cells Dev. 16(4), 547-560 (2007).
11. Soker S, Takashima S, M iao H Q , N eufeld G, Klagsbrun $M$ : N europilin-1 is expressed by endothelial and tumor cells as an isoform-specific receptor for vascular endothelial growth factor. Cell 92(6), 735-745 (1998).

12. Faloon $\mathrm{P}$, Arentson $\mathrm{E}, \mathrm{K}$ azarov $\mathrm{A}$ et al.: Basic fibroblast growth factor positively regulates hematopoietic development. D evelopment 127(9), 1931-1941 (2000).

13. Pearson S, Sroczynska P, Lacaud G Kouskoff $\mathrm{V}$ : The stepwise specification of embryonic stem cells to hematopoietic fate is driven by sequential exposure to Bmp4, activin A, bFGF and VEG F. D evelopment 135(8), 1525-1535 (2008).

14. Pick M, Azzola L, M ossman A, Stanley EG, Elefanty AG : D ifferentiation of human embryonic stem cells in serum-free medium reveals distinct roles for bone morphogenetic protein 4 , vascular endothelial growth factor, stem cell factor, and fibroblast growth factor 2 in hematopoiesis. Stem Cells25(9), 2206-2214 (2007). 
15. M artin M J, M uotri A, Gage F, Varki A: $H$ uman embryonic stem cells express an immunogenic nonhuman sialic acid. N at. M ed. 11(2), 228-232 (2005).

16. Huber TL, Zhou Y, M ead PE, Zon LI: Cooperative effects of growth factors involved in the induction of hematopoietic mesoderm. Blood 92(11), 4128-4137 (1998).

17. Winnier G, Blessing M , Labosky PA, $\mathrm{H}$ ogan BL: Bone morphogenetic protein-4 is required for mesoderm formation and patterning in the mouse. Genes D ev. 9(17), 2105-2116 (1995).

18. Johansson BM , Wiles M V : Evidence for involvement of activin $A$ and bone morphogenetic protein 4 in mammalian mesoderm and hematopoietic development. M ol. Cell Biol. 15(1), 141-151 (1995).

19. Wiles M V, Johansson BM : Embryonic stem cell development in a chemically defined medium. Exp. Cell Res. 247(1), 241-248 (1999).

20. Nakayama N, Lee J, Chiu L: Vascular endothelial growth factor synergistically enhances bone morphogenetic protein-4dependent lymphohematopoietic cell generation from embryonic stem cells in vitro. Blood 95(7), 2275-2283 (2000).
21. Li F, Lu S-J, Vida L, Thomson JA, $H$ onig G R: Bone morphogenetic protein-4 induces efficient hematopoietic differentiation of rhesus monkey embryonic stem cells in vitro. Blood 98(2), 335-342 (2001).

22. Tian X, M orris JK, Linehan JL, Kaufman DS: Cytokine requirements differ for stroma and embryoid body-mediated hematopoiesis from human embryonic stem cells. Exp. H ematol. 32(10), 1000-1009 (2004).

23. Chadwick $K$, Wang $L$, Li $L$ et al.: Cytokines and BM P-4 promote hematopoietic differentiation of human embryonic stem cells. Blood 102(3), 906-915 (2003).

24. Cerdan C, Rouleau A, Bhatia M : VEG F-A165 augments erythropoietic development from human embryonic stem cells. Blood 103(7), 2504-2512 (2004).

25. Park C, Afrikanova I, Chung YS et al.: A hierarchical order of factors in the generation of FLK - and SCL-expressing hematopoietic and endothelial progenitors from embryonic stem cells. D evelopment 131(11), 2749-2762 (2004).

26. Xu C, Rosler E, Jiang J et al.: Basic fibroblast growth factor supports undifferentiated human embryonic stem cell growth without conditioned medium. Stem Cells 23(3), 315-323 (2005)
27. XuRH, Peck RM, Li DS, Feng $X$, Ludwig T, Thomson JA: Basic FGF and suppression of BM $P$ signaling sustain undifferentiated proliferation of human ES cells. $N$ at. M ethods 2(3), 185-190 (2005).

28. Levenstein ME, Ludwig TE, Xu RH et al.: Basic fibroblast growth factor support of human embryonic stem cell self-renewal. Stem Cells 24(3), 568-574 (2006).

29. Yeoh JS, van $O S R$, Weersing $E$ et al.: Fibroblast growth factor- 1 and -2 preserve long-term repopulating ability of hematopoietic stem cells in serum-free cultures. Stem Cells24(6), 1564-1572 (2006).

30. D ell'Era P, Ronca R, Coco L, N icoli S, $M$ etra M , Presta M : Fibroblast growth factor receptor-1 is essential for in vitro cardiomyocyte development. Circ. Res. 93(5), 414-420 (2003).

31. de $H$ ann $G$, Weersing $E$, D ontje $B$ et al.: In vitro generation of long-term repopulating hematopoietic stem cells by fibroblast growth factor-1. D ev. Cell 4(2), 241-251 (2003).

32. Ginis I, Luo Y, M iura T et al.: Differences between human and mouse embryonic stem cells. D ev. Biol. 269(2), 360-380 (2004).

33. D'Amour KA, Agulnick AD, Eliazer $S$, Kelly O G, Kroon E, Baetge EE: Efficient differentiation of human embryonic stem cells to definitiveendoderm. $\mathrm{N}$ at. Biotechnol. 23(12), 1534-1541 (2005). 